\title{
Synthesis and coordination chemistry of an alkyne functionalised bis(pyrazolyl)methane ligand
}

\author{
Fabian Mohr, ${ }^{* a}$ Elena Cerrada ${ }^{b}$ and Mariano Laguna $^{b}$ \\ Received 13th September 2006, Accepted 16th October 2006 \\ First published as an Advance Article on the web 24th October 2006 \\ DOI: 10.1039/b613311h
}

The alkyne functionalised bidentate $\mathrm{N}$-donor ligand (2-propargyloxyphenyl)bis(pyrazolyl)methane (L) was prepared in high yield from the reaction of (2-hydroxyphenyl)bis(pyrazolyl)methane with propargyl bromide in the presence of base. A series of transition-metal complexes including $\left[\mathrm{MCl}_{2} \mathbf{L}\right](\mathbf{M}=\mathrm{Cu}$, $\mathrm{Co}, \mathrm{Ni}, \mathrm{Zn}, \mathrm{Pt}),\left[\mathrm{ML}_{2}\right]\left(\mathrm{NO}_{3}\right)_{2}(\mathrm{M}=\mathrm{Cu}, \mathrm{Co}, \mathrm{Ni}, \mathrm{Zn}),[\mathrm{AgL}] \mathrm{NO}_{3}$ and $[\mathrm{Pd}(\mathbf{L})(\mathrm{dppe})](\mathrm{OTf})_{2}$ were prepared and characterised by spectroscopic techniques. In addition, ligand $\mathbf{L}$ as well as the $\mathrm{Co}$ (II) and $\mathrm{Zn}$ (II) complexes $\left[\mathrm{CoCl}_{2} \mathbf{L}\right]_{2},\left[\mathrm{ZnCl}_{2} \mathbf{L}\right]$ were structurally characterized by single-crystal X-ray diffraction. The organometallic gold(I) and platinum(II) acetylide complexes $\left[\mathrm{Pz}_{2} \mathrm{CH}\left(\mathrm{C}_{6} \mathrm{H}_{4}-2-\mathrm{OCH}_{2} \mathrm{C} \equiv \mathrm{CAuPPh}_{3}\right)\right]$ and trans $-\left[\left\{\mathrm{Pz}_{2} \mathrm{CHC}_{6} \mathrm{H}_{4}-2-\mathrm{OCH}_{2} \mathrm{C} \equiv \mathrm{C}\right\}_{2} \mathrm{Pt}\left(\mathrm{PPh}_{3}\right)_{2}\right]$ were prepared from $\mathbf{L}$ and $\left[\mathrm{AuCl}\left(\mathrm{PPh}_{3}\right)\right]$ and trans- $\left[\mathrm{PtCl}_{2}\left(\mathrm{PPh}_{3}\right)_{2}\right]$, respectively. Treatment of these complexes with $\left[\mathrm{Pd}(\mathrm{OTf})_{2}(\mathrm{dppe})\right]$ or $\left[\mathrm{Cu}(\mathrm{MeCN})_{4}\right] \mathrm{PF}_{6}$ results in formation of the cationic, mixed-metal complexes, which were isolated $(\mathrm{Pt} / \mathrm{Pd}, \mathrm{Au} / \mathrm{Pt})$ or detected by electrospray mass spectrometry $(\mathrm{Au} / \mathrm{Cu}, \mathrm{Pt} / \mathrm{Cu})$.

\section{Introduction}

The large family of bis-and tris(pyrazolyl)borates, commonly referred to as "scorpionate ligands", that were pioneered by Trofinmenko in the 1960s have seen extensive use in modern coordination chemistry. ${ }^{1-5}$ A vast number of transition-metal and main-group metal complexes containing these ligands and their substituted or otherwise modified derivatives have been synthesised and structurally characterised. In contrast, neutral analogues of the scorpionates such as bis- and tris(pyrazolyl)methanes have received considerably less attention. ${ }^{6-8}$ More recently however, a variety of "heteroscorpionate" ligands based on bis(pyrazolyl)methane derivatives containing additional donor atoms such as $\mathrm{O}, \mathrm{N}$ and $\mathrm{S}$ have been reported; some examples are illustrated in Fig 1. ${ }^{5,9}$

In our group with have developed heterometallic gold/silver complexes and have also studied the chemistry of various phosphinegold(I) acetylide complexes. ${ }^{10-13}$ In pursuing our interest in this area of chemistry we wished to design a ligand possessing an alkyne functionality as well as a bidentate N-donor site. Such a ligand should form metal acetylide complexes via the alkyne unit and, at the same time, could be coordinated to different metal centres through the N-donor atoms. For this purpose, alkyne functionalisation of a bis(pyrazolyl)methane derivative seemed the most obvious choice so we began to explore the synthesis and coordination chemistry of such a ligand. The results of our endeavours are reported here.

${ }^{a}$ Fachbereich C, Anorganische Chemie, Bergische Universität Wuppertal, D42119, Wuppertal, Germany. E-mail:fmohr@uni-wuppertal.de; Fax: 49202 439 3053; Tel: 492024393641

${ }^{b}$ Departamento de Química Inorgánica, Instituto de Ciencia de Materiales de Aragón, Universidad de Zaragoza-C.S.I.C., E-50009, Zaragoza, Spain<smiles>O=C(O)C(n1cccn1)n1cccn1</smiles><smiles>S=C(S)C(n1cccn1)n1cccn1</smiles><smiles>OCC(n1cccn1)n1cccn1</smiles><smiles>Oc1ccccc1C(n1cccn1)n1cccn1</smiles><smiles>Sc1ccccc1C(n1cccn1)n1cccn1</smiles><smiles>c1ccc(C(n2cccn2)n2cccn2)nc1</smiles><smiles>Cn1ccnc1C(n1cccn1)n1cccn1</smiles><smiles>c1csc(C(n2cccn2)n2cccn2)c1</smiles>

Fig. 1 Examples of heteroscorpionate pyrazolylmethane ligands.

\section{Experimental}

\section{General}

${ }^{1} \mathrm{H},{ }^{13} \mathrm{C}\left\{{ }^{1} \mathrm{H}\right\}$ and ${ }^{31} \mathrm{P}\left\{{ }^{1} \mathrm{H}\right\}$ NMR spectra were recorded on a $400 \mathrm{MHz}$ Bruker Avance spectrometer. Chemical shifts are quoted relative to external TMS $\left({ }^{1} \mathrm{H},{ }^{13} \mathrm{C}\right)$ and $85 \% \mathrm{H}_{3} \mathrm{PO}_{4}\left({ }^{31} \mathrm{P}\right)$; coupling constants are reported in $\mathrm{Hz}$. FAB mass spectra were measured on a VG Autospec spectrometer using NBA as matrix. Electrospray (ES) mass spectra were recorded on a Bruker Daltonics Microtof$\mathrm{Q}$ instrument as $\mathrm{MeCN}$ solutions. IR spectra were recorded as $\mathrm{KBr}$ disks on a Perkin Elmer Spectrum One instrument. Elemental analyses were obtained in-house using a Perkin Elmer 240B microanalyser. $\left[\mathrm{AuCl}\left(\mathrm{PPh}_{3}\right)\right],{ }^{14}\left[\mathrm{Pd}(\mathrm{OTf})_{2}(\mathrm{dppe})\right]^{15}$ as well as (2-hydroxyphenyl)bis(pyrazolyl)methane ${ }^{16}$ were prepared by 
published procedures. The precursor required for this phenol, bis(pyrazolyl)methanone, was prepared by a modified literature procedure ${ }^{17}$ using bis(trichloromethyl) carbonate ${ }^{18}$ in order to avoid handling and use of highly toxic phosgene. trans$\left[\mathrm{PtCl}_{2}\left(\mathrm{PPh}_{3}\right)_{2}\right]$ was prepared by the reaction of two equivalents of $\mathrm{PPh}_{3}$ with $\left[\mathrm{PtCl}_{2}(\mathrm{tht})_{2}\right]$ (tht $=$ tetrahydrothiophene). All other reagents and solvents were sourced commercially and used as received.

\section{Preparations}

(2-Propargyloxyphenyl)bis(pyrazolyl)methane (L). A mixture of (2-hydroxyphenyl)bis(pyrazolyl)methane $(2.5 \mathrm{~g}, 0.010 \mathrm{~mol})$, $\mathrm{K}_{2} \mathrm{CO}_{3}$ (4.3 g, $\left.0.031 \mathrm{~mol}\right)$ and propargyl bromide $(1.7 \mathrm{~mL}$, $0.019 \mathrm{~mol})$ in acetone $(80 \mathrm{~mL})$ was refluxed for $8 \mathrm{~h}$. The solution was filtered and the filtrate evaporated to dryness affording $2.7 \mathrm{~g}$ $(92 \%)$ of pale yellow microcrystalline solid. ${ }^{1} \mathrm{H}$ NMR $(\delta$ in acetone$\left.\mathrm{d}_{6}\right): 3.08(\mathrm{t}, J=2.5 \mathrm{~Hz}, 1 \mathrm{H}, \equiv \mathrm{CH}), 4.78(\mathrm{~d}, J=2.3 \mathrm{~Hz}, 2 \mathrm{H}$, $\left.\mathrm{OCH}_{2}\right), 6.31(\mathrm{dd}, J=2.7,2.0 \mathrm{~Hz}, 2 \mathrm{H}, \mathrm{Pz}-H 4), 6.98(\mathrm{dt}, J=$ 6.3, $\left.0.5 \mathrm{~Hz}, 1 \mathrm{H}, H 5^{\prime}\right), 7.03\left(\mathrm{dd}, J=7.8,0.5 \mathrm{~Hz}, 1 \mathrm{H}, H 6^{\prime}\right), 7.21$ $\left(\mathrm{d}, J=8.3,1 \mathrm{H}, H 3^{\prime}\right), 7.43\left(\mathrm{dt}, J=8.4,2.0 \mathrm{~Hz}, 1 \mathrm{H}, H 4^{\prime}\right), 7.54$ (d, $J=1.8 \mathrm{~Hz}, 2 \mathrm{H}, \mathrm{Pz}-H 3), 7.59$ (d, $J=2.5 \mathrm{~Hz}, 2 \mathrm{H}, \mathrm{Pz}-H 5)$, $8.03(\mathrm{~s}, 1 \mathrm{H}, \mathrm{CH}) .{ }^{13} \mathrm{C}\left\{{ }^{1} \mathrm{H}\right\}$ NMR $\left(\delta\right.$ in acetone-d $\left.{ }_{6}\right): 57.91\left(\mathrm{OCH}_{2}\right)$, $74.63(\mathrm{CH}), 78.42(\equiv C \mathrm{H}), 80.20(C \equiv \mathrm{CH}), 107.49(\mathrm{Pz}-C 4), 114.59$ $\left(C 3^{\prime}\right), 123.07\left(C 5^{\prime}\right), 127.22\left(C 1^{\prime}\right), 129.94\left(C 6^{\prime}\right), 131.31(\mathrm{Pz}-C 5)$, $132.34\left(C 4^{\prime}\right), 141.75(\mathrm{Pz}-C 3), 156.65\left(C 2^{\prime}\right) . \mathrm{IR}\left(\mathrm{cm}^{-1} \mathrm{KBr}\right): 3208$ $v(\mathrm{C} \equiv \mathrm{C}-\mathrm{H}), 2124 v(\mathrm{C} \equiv \mathrm{C})$. FAB-MS $m / z: 279[\mathrm{M}]^{+}, 223[\mathrm{M}-$ $\left.\mathrm{OCH}_{2} \mathrm{C} \equiv \mathrm{CH}\right]^{+}, 211[\mathrm{M}-\mathrm{Pz}]^{+}$. Anal. Calc. for $\mathrm{C}_{16} \mathrm{H}_{14} \mathrm{~N}_{4} \mathrm{O}: \mathrm{C}$, 69.05; H, 5.07; N, 20.13. Found: C, 68.88; H, 4.90; N, 20.37\%. $\mathrm{X}$-Ray quality crystals were obtained by slow evaporation of an EtOH solution of the compound.

$\left[\mathbf{M L}_{2}\right] \mathbf{X}$ complexes. To a solution of the metal nitrate in EtOH $(10 \mathrm{~mL})$ was added a solution of $\mathbf{L}$ in acetone $(10 \mathrm{~mL})$. The mixture was left to stand in an open vessel and crystals of the complex deposited after a few days. The solids were collected on a frit, washed with small amounts of $\mathrm{EtOH}$ and $\mathrm{Et}_{2} \mathrm{O}$ and dried in vacuum.

$\left[\mathrm{CuL}_{2}\right]\left(\mathrm{NO}_{3}\right)_{2}$ (1). Blue solid 92\% yield. IR ( $\left.\mathrm{cm}^{-1} \mathrm{KBr}\right): 3242$ $v(\mathrm{C} \equiv \mathrm{C}-\mathrm{H}), 2118 v(\mathrm{C} \equiv \mathrm{C}) ; \mathrm{ES}-\mathrm{MS} m / z: 619\left[\mathrm{M}-2 \mathrm{NO}_{3}\right]^{+}, 341$ $\left[\mathrm{M}-\mathrm{L}-2 \mathrm{NO}_{3}\right]^{+}$. Anal. Calc. for $\mathrm{C}_{32} \mathrm{H}_{28} \mathrm{~N}_{10} \mathrm{O}_{8} \mathrm{Cu} \cdot 2 \mathrm{H}_{2} \mathrm{O}: \mathrm{C}, 49.26$; H, 4.13; N, 17.95. Found: C, 49.30; H, 3.83; N, 18.18\%.

[NiL $\mathbf{N}$ ( $\left.\mathbf{N O}_{3}\right)_{2}$ (2). Blue solid 83\% yield. IR $\left(\mathrm{cm}^{-1} \mathrm{KBr}\right): 3284$ $v(\mathrm{C} \equiv \mathrm{C}-\mathrm{H}), 2119 v(\mathrm{C} \equiv \mathrm{C}) ; \mathrm{ES}-\mathrm{MS} m / z$ : $676\left[\mathrm{M}-\mathrm{NO}_{3}\right]^{+}, 398[\mathrm{M}-$ $\left.\mathrm{L}-\mathrm{NO}_{3}\right]^{+}$. Anal. Calc. for $\mathrm{C}_{32} \mathrm{H}_{28} \mathrm{~N}_{10} \mathrm{O}_{8} \mathrm{Ni}$ : C, 51.99; H, 3.82; N, 18.95. Found: C, 51.69; H, 3.79; N, 18.61\%.

$\left[\mathrm{CoL}_{2}\right]\left(\mathrm{NO}_{3}\right)_{2}$ (3). Pink solid 86\% yield. IR ( $\left.\mathrm{cm}^{-1} \mathrm{KBr}\right): 3283$ $v(\mathrm{C} \equiv \mathrm{C}-\mathrm{H}), 2119 v(\mathrm{C} \equiv \mathrm{C}) . \mathrm{ES}-\mathrm{MS} m / z: 677\left[\mathrm{M}-\mathrm{NO}_{3}\right]^{+}, 399[\mathrm{M}-$ $\left.\mathrm{L}-\mathrm{NO}_{3}\right]^{+}$. Anal. Calc. for $\mathrm{C}_{32} \mathrm{H}_{28} \mathrm{~N}_{10} \mathrm{O}_{8} \mathrm{Co}$ : C, 51.97; H, 3.82; N, 18.94. Found: C, 51.70; H, 3.77; N, 18.53\%.

$\left[\mathrm{ZnL}_{2}\right]\left(\mathrm{NO}_{3}\right)_{2}$ (4). Colourless solid $85 \%$ yield. ${ }^{1} \mathrm{H}$ NMR $(\delta$ in acetone- $\left.\mathrm{d}_{6}\right): 3.13(\mathrm{t}, J=2.3 \mathrm{~Hz}, 2 \mathrm{H}, \equiv \mathrm{CH}), 4.80(\mathrm{~d}, J=2.3 \mathrm{~Hz}$, $4 \mathrm{H}, \mathrm{OCH}_{2}$ ), 6.51 (br s, $\left.4 \mathrm{H}, \mathrm{Pz}-H 4\right), 6.83$ (d, $J=7.8 \mathrm{~Hz}, 2 \mathrm{H}$, $\left.H 6^{\prime}\right), 6.95$ (t, $\left.J=7.6 \mathrm{~Hz}, 2 \mathrm{H}, H 5^{\prime}\right), 7.18(\mathrm{~d}, J=8.3 \mathrm{~Hz}, 2 \mathrm{H}$, $\left.H 3^{\prime}\right), 7.42$ (dt, $\left.J=8.8,1.8 \mathrm{~Hz}, 2 \mathrm{H}, H 4^{\prime}\right), 7.78$ (br s, $4 \mathrm{H}, \mathrm{Pz}-H 3$ ), 8.06 (br s, 4 H, Pz-H5), 8.36 (s, 2 H, CH). IR ( $\mathrm{cm}^{-1} \mathrm{KBr}$ ): 3279 $v(\mathrm{C} \equiv \mathrm{C}-\mathrm{H}), 2119 v(\mathrm{C} \equiv \mathrm{C}) . \mathrm{ES}-\mathrm{MS} m / z: 682\left[\mathrm{M}-\mathrm{NO}_{3}\right]^{+}, 342[\mathrm{M}-$
$\left.\mathrm{L}-2 \mathrm{NO}_{3}\right]^{+}$. Anal. Calc. for $\mathrm{C}_{32} \mathrm{H}_{28} \mathrm{~N}_{10} \mathrm{O}_{8} \mathrm{Zn}: \mathrm{C}, 51.52 ; \mathrm{H}, 3.78 ; \mathrm{N}$, 18.78. Found: C, 51.59; H, 3.82; N, 18.81\%.

$\left[\mathrm{AgL}_{2} \mathbf{N O}_{3}\right.$ (5). Colourless solid 79\% yield. ${ }^{1} \mathrm{H}$ NMR $(\delta$ in acetone- $\left.\mathrm{d}_{6}\right): 3.09(\mathrm{t}, J=2.5 \mathrm{~Hz}, 2 \mathrm{H}, \equiv \mathrm{CH}), 4.74(\mathrm{~d}, J=1.8 \mathrm{~Hz}$, $\left.4 \mathrm{H}, \mathrm{OCH}_{2}\right), 6.38(\mathrm{t}, J=2.0 \mathrm{~Hz}, 4 \mathrm{H}, \mathrm{Pz}-H 4), 6.98-7.01(\mathrm{~m}, 4$ $\left.\mathrm{H}, H 5^{\prime}, H 6^{\prime}\right), 7.21\left(\mathrm{~d}, J=8.3 \mathrm{~Hz}, 2 \mathrm{H}, H 3^{\prime}\right), 7.44$ (dt, $J=8.8$, $\left.4.5 \mathrm{~Hz}, 2 \mathrm{H}, H 4^{\prime}\right), 7.55$ (d, $\left.J=1.8 \mathrm{~Hz}, 4 \mathrm{H}, \mathrm{Pz}-H 3\right), 7.87$ (d, $J=2.5 \mathrm{~Hz}, 4 \mathrm{H}, \mathrm{Pz}-H 5), 8.18$ (s, $2 \mathrm{H}, \mathrm{CH}) . \mathrm{IR}\left(\mathrm{cm}^{-1} \mathrm{KBr}\right): 3210$ $v(\mathrm{C} \equiv \mathrm{C}-\mathrm{H}), 2124 v(\mathrm{C} \equiv \mathrm{C}) . \mathrm{ES}-\mathrm{MS} m / z: 663\left[\mathrm{M}-\mathrm{NO}_{3}\right]^{+}, 385[\mathrm{M}-$ $\left.\mathrm{L}-\mathrm{NO}_{3}\right]^{+}$. Anal. Calc. for $\mathrm{C}_{32} \mathrm{H}_{28} \mathrm{~N}_{9} \mathrm{O}_{5} \mathrm{Ag}: \mathrm{C}, 52.90 ; \mathrm{H}, 3.88 ; \mathrm{N}$, 17.35. Found: C, 52.69; H, 3.78; N, 17.16\%.

$\left[\mathbf{M C l}_{2} \mathbf{L}\right]$ complexes. To a solution of the metal chloride in EtOH $(10 \mathrm{~mL})$ was added an equimolar amount of $\mathbf{L}$ dissolved in acetone $(10 \mathrm{~mL})$. The mixture was left to stand in an open vessel and crystals of the complex deposited after a few days. In the case of the $\mathrm{Cu}$ derivative, a greenish solid precipitated out instantaneously. The solid was collected on a frit, washed with small amounts of $\mathrm{EtOH}$ and $\mathrm{Et}_{2} \mathrm{O}$ and dried in vacuum.

[CuCl$\left.{ }_{2} \mathbf{L}\right]$ (6). Light green solid 79\% yield. IR ( $\left.\mathrm{cm}^{-1} \mathrm{KBr}\right): 3238$ $v(\mathrm{C} \equiv \mathrm{C}-\mathrm{H}), 2115 v(\mathrm{C} \equiv \mathrm{C}) . \mathrm{FAB}-\mathrm{MS} m / z: 754\left[\mathrm{M}_{2}\right]^{+}, 376[\mathrm{M}-\mathrm{Cl}]^{+}$, $341[\mathrm{M}-2 \mathrm{Cl}]^{+}$. Anal. Calc. for $\mathrm{C}_{32} \mathrm{H}_{28} \mathrm{~N}_{8} \mathrm{O}_{2} \mathrm{Cl}_{4} \mathrm{Cu}_{2}$ : C, 46.56; $\mathrm{H}$, 3.42; N, 13.57. Found: C, 46.68; H, 3.30; N, 13.46\%.

[ $\left.\mathbf{N i C l}_{2} \mathbf{L}\right]$ (7). Green solid 63\% yield. IR ( $\left.\mathrm{cm}^{-1} \mathrm{KBr}\right): 2117$ $v(\mathrm{C} \equiv \mathrm{C})$. FAB-MS m/z: $779\left[\mathrm{M}_{2}-\mathrm{Cl}\right]^{+}, 371[\mathrm{M}-\mathrm{Cl}]^{+}, 336[\mathrm{M}-$ $2 \mathrm{Cl}]^{+}$. Anal. Calc. for $\mathrm{C}_{32} \mathrm{H}_{28} \mathrm{~N}_{8} \mathrm{O}_{2} \mathrm{Cl}_{4} \mathrm{Ni}_{2}: \mathrm{C}, 47.11 ; \mathrm{H}, 3.46 ; \mathrm{N}$, 13.74. Found: C, 47.45; H, 3.27; N, 13.70\%.

$\left[\mathrm{CoCl}_{2} \mathbf{L}\right]_{2}$ (8). Bright blue solid $81 \%$ yield. IR $\left(\mathrm{cm}^{-1}\right.$ $\mathrm{KBr}): 3258 \quad v(\mathrm{C} \equiv \mathrm{C}-\mathrm{H}), \quad 2116 \quad v(\mathrm{C} \equiv \mathrm{C})$. Anal. Calc. for $\mathrm{C}_{32} \mathrm{H}_{28} \mathrm{~N}_{8} \mathrm{O}_{2} \mathrm{Cl}_{4} \mathrm{Co}_{2}$ : C, 47.08; $\mathrm{H}, 3.46 ; \mathrm{N}, 13.73$. Found: $\mathrm{C}, 46.91$; $\mathrm{H}, 3.20$; N, $13.58 \%$. An X-ray quality crystal was selected from the bulk sample.

[ $\left.\mathbf{Z n C l}_{2} \mathbf{L}\right]$ (9). Colourless solid $70 \%$ yield. ${ }^{1} \mathrm{H}$ NMR ( $\delta$ in acetone- $\left.\mathrm{d}_{6}\right): 3.15(\mathrm{t}, J=2.5 \mathrm{~Hz}, 1 \mathrm{H}, \equiv \mathrm{CH}), 4.84(\mathrm{~d}, J=2.3 \mathrm{~Hz}$, $\left.2 \mathrm{H}, \mathrm{OCH}_{2}\right), 6.57(\mathrm{t}, J=2.5 \mathrm{~Hz}, 2 \mathrm{H}, \mathrm{Pz}-H 4), 7.02$ (dt, $J=7.8$, $\left.1.0 \mathrm{~Hz}, 1 \mathrm{H}, H 5^{\prime}\right), 7.09$ (dd, $\left.J=7.8,1.5 \mathrm{~Hz}, 1 \mathrm{H}, H 6^{\prime}\right), 7.19$ (dd, $\left.J=8.3,0.5 \mathrm{~Hz}, 1 \mathrm{H}, H 3^{\prime}\right), 7.45\left(\mathrm{dt}, J=8.3,1.8 \mathrm{~Hz}, 1 \mathrm{H}, H 4^{\prime}\right)$, 7.91 (d, $J=1.8 \mathrm{~Hz}, 2 \mathrm{H}, \mathrm{Pz}-H 3), 8.18$ (br s, $2 \mathrm{H}, \mathrm{Pz}-H 5), 8.48$ (s, $2 \mathrm{H}, \mathrm{CH})$. IR $\left(\mathrm{cm}^{-1} \mathrm{KBr}\right): 3258 v(\mathrm{C} \equiv \mathrm{C}-\mathrm{H}), 2114 v(\mathrm{C} \equiv \mathrm{C})$. Anal. Calc. for $\mathrm{C}_{32} \mathrm{H}_{28} \mathrm{~N}_{8} \mathrm{O}_{2} \mathrm{Cl}_{4} \mathrm{Zn}_{2}$ : C, 46.35; H, 3.40; N, 13.51. Found: C, 46.20; H, 3.36; N, 13.27\%. An X-ray quality crystal was selected from the bulk sample.

$[\mathbf{P d}(\mathbf{L})(\mathbf{d p p e})](\mathbf{O T f})_{2} \quad$ (10). To a $\quad \mathrm{CH}_{2} \mathrm{Cl}_{2}$ solution of $\left[\mathrm{Pd}(\mathrm{OTf})_{2}\right.$ (dppe) $](0.144 \mathrm{~g}, 0.179 \mathrm{mmol})$ was added a solution of L $(0.050 \mathrm{~g}, 0.179 \mathrm{mmol})$ in the same solvent. After stirring for $4 \mathrm{~h}$ the mixture was passed through Celite and the filtrate was concentrated in vacuum. Addition of pentane gave a pale-yellow solid which was isolated by filtration and dried. Yield $86 \%{ }^{1} \mathrm{H}$ NMR $\left(\delta\right.$ in $\left.\mathrm{CDCl}_{3}\right): 2.42(\mathrm{t}, J=2.3 \mathrm{~Hz}, 1 \mathrm{H}, \equiv \mathrm{CH}), 2.73$ (br s, $2 \mathrm{H}, \mathrm{PCH}_{2} \mathrm{CH}_{2} \mathrm{P}$ ), 3.16 (br s, $2 \mathrm{H}, \mathrm{PCH}_{2} \mathrm{CH}_{2} \mathrm{P}$ ), 4.62 (d, $J=$ $\left.2.3 \mathrm{~Hz}, 2 \mathrm{H}, \mathrm{OCH}_{2}\right), 6.05\left(\mathrm{~d}, J=7.3 \mathrm{~Hz}, 1 \mathrm{H}, H 6^{\prime}\right), 6.28$ (t, $J=$ $2.3 \mathrm{~Hz}, 2 \mathrm{H}, \mathrm{Pz}-H 4), 6.42$ (t, $\left.J=7.3 \mathrm{~Hz}, 1 \mathrm{H}, H 5^{\prime}\right), 6.88$ (d, $J=$ $2.3 \mathrm{~Hz}, 2 \mathrm{H}, \mathrm{Pz}-H 3), 7.15\left(\mathrm{~d}, J=8,1 \mathrm{~Hz}, 1 \mathrm{H}, H 3^{\prime}\right), 7.25-7.32$ (m, $4 \mathrm{H}, \mathrm{Ph} 2 \mathrm{P}), 7.41$ (dt, $\left.J=8.6,1.2 \mathrm{~Hz}, 1 \mathrm{H}, H 4^{\prime}\right), 7.45-7.97$ $\left(\mathrm{m}, 16 \mathrm{H}, \mathrm{Ph}_{2} \mathrm{P}\right), 8.64$ (d, $\left.J=1.2 \mathrm{~Hz}, 2 \mathrm{H}, \mathrm{Pz}-H 5\right), 8.89$ (s, 1 $\mathrm{H}, \mathrm{CH}) .{ }^{31} \mathrm{P}$ NMR $\left(\delta\right.$ in $\left.\mathrm{CDCl}_{3}\right): 68.54$. IR $\left(\mathrm{cm}^{-1} \mathrm{KBr}\right): 3245$ 
$v(\mathrm{C} \equiv \mathrm{C}-\mathrm{H}), 2121 v(\mathrm{C} \equiv \mathrm{C}), 1264 v\left(\mathrm{SO}_{3}\right), 1157 v\left(\mathrm{CF}_{3}\right)$. FAB-MS $m / z: 782[\mathrm{M}]^{+}, 653\left[\mathrm{M}-\mathrm{C}_{6} \mathrm{H}_{4} \mathrm{OCH}_{2} \mathrm{CCH}\right]^{+}, 504[\mathrm{M}-\mathrm{L}]^{+}$. Anal. Calc. for $\mathrm{C}_{44} \mathrm{H}_{38} \mathrm{~N}_{4} \mathrm{O}_{7} \mathrm{P}_{2} \mathrm{~S}_{2} \mathrm{~F}_{6} \mathrm{Pd}$ : C, 48.87; H, 3.54; N, 5.18. Found: C, 48.77; H, 3.43; N, 5.17\%.

$\left[\mathrm{Pz}_{2} \mathbf{C H}\left(\mathrm{C}_{6} \mathrm{H}_{4}-2-\mathrm{OCH}_{2} \mathrm{CCAuPPh}_{3}\right)\right]$ (11). A mixture of $\mathbf{L}$ (0.309 g, $1.11 \mathrm{mmol}),\left[\mathrm{AuCl}\left(\mathrm{PPh}_{3}\right)\right](0.500 \mathrm{~g}, 1.01 \mathrm{mmol})$ and $\mathrm{KOH}$ $(0.091 \mathrm{~g}, 1.62 \mathrm{mmol})$ in $\mathrm{MeOH}(50 \mathrm{~mL})$ was stirred for $c a$. $18 \mathrm{~h}$. The orange solution was evaporated to dryness and the resulting solid extracted into $\mathrm{CH}_{2} \mathrm{Cl}_{2}$. The $\mathrm{CH}_{2} \mathrm{Cl}_{2}$ extracts were passed through Celite and concentrated in vacuum. Addition of $\mathrm{Et}_{2} \mathrm{O}$ precipitated a pale yellow solid which was isolated by filtration and dried. Yield: $0.682 \mathrm{~g}, 92 \% .{ }^{1} \mathrm{H}$ NMR $\left(\delta\right.$ in $\left.\mathrm{CDCl}_{3}\right): 4.81(\mathrm{~d}, J=1.5 \mathrm{~Hz}, 2 \mathrm{H}$, $\left.\mathrm{OCH}_{2}\right), 6.26$ (t, $\left.J=2.0 \mathrm{~Hz}, 2 \mathrm{H}, \mathrm{Pz}-H 4\right), 6.92-7.00$ (m, $2 \mathrm{H}, H 5^{\prime}$, $\left.H 6^{\prime}\right), 7.29\left(\mathrm{~d}, J=8.3 \mathrm{~Hz}, 1 \mathrm{H}, H 3^{\prime}\right), 7.37(\mathrm{dt}, J=8.6,2.0 \mathrm{~Hz}$, $\left.1 \mathrm{H}, H 4^{\prime}\right), 7.41-7.55$ (m, $\left.17 \mathrm{H}, \mathrm{Ph}_{3} \mathrm{P}, \mathrm{Pz}-H 5\right), 7.60$ (d, $J=1.5 \mathrm{~Hz}$, $2 \mathrm{H}, \mathrm{Pz}-H 3), 8.04$ (s, $1 \mathrm{H}, \mathrm{CH}) .{ }^{31} \mathrm{P} \mathrm{NMR}\left(\delta\right.$ in $\left.\mathrm{CDCl}_{3}\right)$ : 42.04. IR $\left(\mathrm{cm}^{-1} \mathrm{KBr}\right): 2135 v(\mathrm{C} \equiv \mathrm{C})$. FAB-MS m/z: $737[\mathrm{M}]^{+}$. Anal. Calc. for $\mathrm{C}_{34} \mathrm{H}_{28} \mathrm{~N}_{4} \mathrm{OPAu}$ : C, 55.44; H, 3.83; N, 7.61. Found: C, 55.54; $\mathrm{H}, 3.91 ; \mathrm{N}, 7.77 \%$.

trans- $\left[\left\{\mathrm{Pz}_{2} \mathrm{CHC}_{6} \mathrm{H}_{4}-2-\mathrm{OCH}_{2} \mathrm{CC}\right\}_{2} \mathbf{P t}\left(\mathrm{PPh}_{3}\right)_{2}\right]$ (12). A mixture of $\mathbf{L}(0.089 \mathrm{~g}, 0.32 \mathrm{mmol})$, trans $-\left[\mathrm{PtCl}_{2}\left(\mathrm{PPh}_{3}\right)_{2}\right](0.115 \mathrm{~g}$, $0.15 \mathrm{mmol}), \mathrm{CuI}(5 \mathrm{mg}), \mathrm{EtOH}(10 \mathrm{~mL})$ and $\mathrm{Et}_{2} \mathrm{NH}(10 \mathrm{~mL})$ was refluxed for $1 \mathrm{~h}$. After cooling to room temperature, the colourless solid was isolated by filtration and washed with $\mathrm{EtOH}$ and $\mathrm{Et}_{2} \mathrm{O}$. Yield: $0.141 \mathrm{~g} 76 \%{ }^{1} \mathrm{H}$ NMR $\left(\delta\right.$ in $\left.\mathrm{CDCl}_{3}\right): 3.91(\mathrm{~s}, 4 \mathrm{H}$, $\left.\mathrm{OCH}_{2}\right), 6.15(\mathrm{t}, J=2.3 \mathrm{~Hz}, 4 \mathrm{H}, \mathrm{Pz}-H 4), 6.44(\mathrm{~d}, J=8.1 \mathrm{~Hz}, 2$ $\left.\mathrm{H}, H 3^{\prime}\right), 6.75-6.85\left(\mathrm{~m}, 4 \mathrm{H}, H 5^{\prime}, H 6^{\prime}\right), 6.90(\mathrm{dt}, J=8.8,1.8 \mathrm{~Hz}$, $\left.2 \mathrm{H}, H 4^{\prime}\right), 7.19(\mathrm{~d}, J=2.3 \mathrm{~Hz}, 4 \mathrm{H}, \mathrm{Pz}-H 3), 7.22-7.28(\mathrm{~m}, 12 \mathrm{H}$, $m-\mathrm{Ph}_{3} \mathrm{P}$ ), 7.32-7.38 (m, $\left.6 \mathrm{H}, p-\mathrm{Ph}_{3} \mathrm{P}\right), 7.56(\mathrm{~d}, J=1.8 \mathrm{~Hz}, 4 \mathrm{H}, \mathrm{Pz}-$ H5), 7.61-7.68 (m, $\left.12 \mathrm{H}, o-\mathrm{Ph}_{3} \mathrm{P}\right), 7.70(\mathrm{~s}, 2 \mathrm{H}, \mathrm{CH}) .{ }^{31} \mathrm{P} \mathrm{NMR}(\delta$ in $\left.\mathrm{CDCl}_{3}\right)$ : $18.91\left(J_{\mathrm{Pt}-\mathrm{P}}=2636 \mathrm{~Hz}\right)$. IR $\left(\mathrm{cm}^{-1} \mathrm{KBr}\right): 2132 v(\mathrm{C} \equiv \mathrm{C})$. FAB-MS $m / z$ : $1206[\mathrm{M}-\mathrm{Pz}]^{+}$. Anal. Calc. for $\mathrm{C}_{68} \mathrm{H}_{56} \mathrm{~N}_{8} \mathrm{O}_{2} \mathrm{P}_{2} \mathrm{Pt}$ : C, 64.09; H, 4.43; N, 8.79. Found: C, 64.32; H, 4.40; N, 8.81\%.

[(dppe) $\left.\mathrm{PdPz}_{2} \mathrm{CH}\left(\mathrm{C}_{6} \mathrm{H}_{4}-\mathbf{2}-\mathrm{OCH}_{2} \mathrm{CCAuPPh}_{3}\right)\right](\mathrm{OTf})_{2}$ (13). To a solution of $11(0.025 \mathrm{~g}, 0.034 \mathrm{mmol})$ in $\mathrm{CH}_{2} \mathrm{Cl}_{2}(10 \mathrm{~mL})$ was added $\left[\mathrm{Pd}(\mathrm{OTf})_{2}(\mathrm{dppe})\right](0.027 \mathrm{~g}, 0.034 \mathrm{mmol})$. After stirring the solution for $2 \mathrm{~h}$ most of the solvent was evaporated and pentane was added. The resulting ochre-yellow precipitate was isolated by filtration and washed with pentane and dried. Yield: $0.041 \mathrm{~g}$, $79 \%$. ${ }^{1} \mathrm{H}$ NMR ( $\delta$ in $\mathrm{CDCl}_{3}$ ): 2.78 (br s, $2 \mathrm{H}, \mathrm{PCH}_{2} \mathrm{CH}_{2} \mathrm{P}$ ), 3.17 (br s, $\left.2 \mathrm{H}, \mathrm{PCH}_{2} \mathrm{CH}_{2} \mathrm{P}\right), 4.84\left(\mathrm{~s}, 2 \mathrm{H}, \mathrm{OCH}_{2}\right), 5.92(\mathrm{~d}, J=7.3 \mathrm{~Hz}$, $\left.1 \mathrm{H}, H 6^{\prime}\right), 6.28$ (br s, $\left.2 \mathrm{H}, \mathrm{Pz}-H 4\right), 6.36$ (t, $J=7.3 \mathrm{~Hz}, 1 \mathrm{H}$, $\left.H 5^{\prime}\right), 6.79$ (br s, $\left.1 \mathrm{H}, \mathrm{Pz}-H 3\right), 7.12$ (d, $\left.J=8,1 \mathrm{~Hz}, 1 \mathrm{H}, H 3^{\prime}\right)$, 7.31-8.08 (m, $36 \mathrm{H}, \mathrm{Ph}_{2} \mathrm{P}, \mathrm{Ph}_{3} \mathrm{P}, H 4^{\prime}$ ), 8.64 (br s, $\left.2 \mathrm{H}, \mathrm{Pz}-H 5\right)$, $8.81(\mathrm{~s}, 1 \mathrm{H}, \mathrm{CH}) \cdot{ }^{31} \mathrm{P}$ NMR $\left(\delta\right.$ in $\left.\mathrm{CDCl}_{3}\right): 45.10\left(\mathrm{~s}, P \mathrm{Ph}_{3}\right), 67.63$ $\left(\mathrm{Ph}_{2} P_{C_{2}} \mathrm{CH}_{2} P \mathrm{Ph}_{2}\right)$. IR $\left(\mathrm{cm}^{-1} \mathrm{KBr}\right): 2200 v(\mathrm{C} \equiv \mathrm{C}), 1259 v\left(\mathrm{SO}_{3}\right)$, $1156 v\left(\mathrm{CF}_{3}\right)$. Anal. Calc. for $\mathrm{C}_{62} \mathrm{H}_{52} \mathrm{~N}_{4} \mathrm{O}_{7} \mathrm{P}_{3} \mathrm{~F} 6 \mathrm{~S}_{2} \mathrm{AuPd}$ : C, 48.37; H, 3.40; N, 3.64. Found: C, 48.54; H, 3.71; N, 3.77\%.

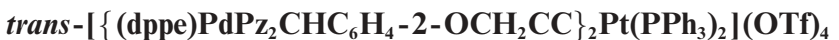
(14). To a solution of $12(0.025 \mathrm{~g}, 0.0196 \mathrm{mmol})$ in $\mathrm{CH}_{2} \mathrm{Cl}_{2}$ $(10 \mathrm{~mL})$ was added $\left[\mathrm{Pd}(\mathrm{OTf})_{2}(\mathrm{dppe})\right](0.032 \mathrm{~g}, 0.0398 \mathrm{mmol})$. After stirring the solution for $2 \mathrm{~h}$ most of the solvent was evaporated and $\mathrm{Et}_{2} \mathrm{O}$ was added. The resulting pale-yellow precipitate was isolated by filtration and washed with $\mathrm{Et}_{2} \mathrm{O}$ and dried. Yield: $0.052 \mathrm{~g}, 93 \%$. ${ }^{1} \mathrm{H}$ NMR ( $\delta$ in acetone- $\mathrm{d}_{6}$ ): 2.91 (br s, $4 \mathrm{H}, \mathrm{PCH}_{2} \mathrm{CH}_{2} \mathrm{P}$ ), 3.31 (br s, $4 \mathrm{H}, \mathrm{PCH}_{2} \mathrm{CH}_{2} \mathrm{P}$ ), 3.67 (s, $\left.4 \mathrm{H}, \mathrm{OCH}_{2}\right), 5.99$ (d, $J=7.3 \mathrm{~Hz}, 2 \mathrm{H}$, $H 3^{\prime}$ ), 6.38 (br s, 4 H, Pz-H4), 6.53-6.61 (m, 4 H, H5', H6'), 7.37- $7.83\left(\mathrm{~m}, 52 \mathrm{H}, \mathrm{Ph}_{2} \mathrm{P}, m-\mathrm{Ph}_{3} \mathrm{P}\right), 7.86-7.94\left(\mathrm{~m}, 6 \mathrm{H}, p-\mathrm{PPh}_{3}\right), 8.26$ (s, $2 \mathrm{H}, \mathrm{CH}), 8.27-8.33\left(\mathrm{~m}, 16 \mathrm{H}, o-\mathrm{Ph}_{3} \mathrm{P}, \mathrm{Pz}-H 3\right) .{ }^{31} \mathrm{P} \mathrm{NMR}(\delta$ in $\left.\mathrm{CDCl}_{3}\right): 73.88\left(\mathrm{~s},\left(\mathrm{Ph}_{2} P \mathrm{PCH}_{2} \mathrm{CH}_{2} P \mathrm{Ph}_{2}\right), 24.01\left(J_{\mathrm{Pt} \mathrm{P}}=2620 \mathrm{~Hz}\right)\right.$. IR $\left(\mathrm{cm}^{-1} \mathrm{KBr}\right): 2126 v(\mathrm{C} \equiv \mathrm{C}), 1259 v\left(\mathrm{SO}_{3}\right), 1155 v\left(\mathrm{CF}_{3}\right)$. Anal. Calc. for $\mathrm{C}_{124} \mathrm{H}_{104} \mathrm{~N}_{8} \mathrm{O}_{14} \mathrm{P}_{6} \mathrm{~F}_{12} \mathrm{~S}_{4} \mathrm{Pd}_{2} \mathrm{Pt}$ : C, 51.71; H, 3.64; N, 3.89. Found: C, 51.91; H, 3.69; N, 3.86\%.

Copper(I) coordination of 11 and 12. Solutions of complexes 11 and 12 in $\mathrm{MeCN}(c a .3 \mathrm{ml})$ were treated with $\left[\mathrm{Cu}(\mathrm{MeCN})_{4}\right] \mathrm{PF}_{6}$ and the resulting solutions were analysed by HR-ES mass spectrometry.

\section{X-Ray crystallography}

Crystals of $\mathbf{L}, \mathbf{8}$ and $\mathbf{9}$ were mounted in oil on a glass fibre and data were collected at $100 \mathrm{~K}$ on either Oxford Diffraction XCalibur2 or Bruker APEX CCD diffractometers. Data were reduced and absorption corrections applied using CrysalisRED, ${ }^{19}$ SAINT $^{20}$ and SADABS. ${ }^{21}$ The structures were solved by direct methods and refined to $F_{\mathrm{o}}{ }^{2}$ using full-matrix least squares. ${ }^{22}$ Hydrogen atoms were placed at calculated positions and refined as riding on their respective carbon atoms. The hydrogen atoms in $\mathbf{8}$ were located on the difference map and refined isotropically. The crystallographic and refinement details are listed in Table 1.

CCDC reference numbers 620814-620816.

For crystallographic data in CIF or other electronic format see DOI: $10.1039 / \mathrm{b} 613311 \mathrm{~h}$

\section{Results and discussion}

\section{Ligand synthesis}

The alkyne functionalised scorpionate ligand (2-propargyloxyphenyl)bis(pyrazolyl)methane (L) was easily prepared in very high yields from the reaction of (2-hydroxyphenyl)bis(pyrazolyl)methane ${ }^{16}$ with propargyl bromide and $\mathrm{K}_{2} \mathrm{CO}_{3}$ in refluxing acetone (Scheme 1). We wish to point out here that ligand L should be considered a "true" scorpionate, since the three donor sites (pyrazolyl and alkyne units) resemble the claws and the stinger of a scorpion much closer than the tris(pyrazolyl)methanes or borates.
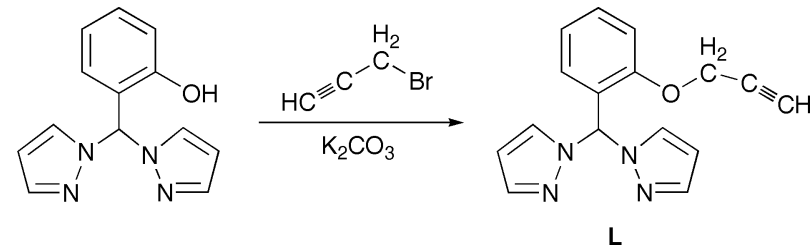

Scheme 1

All spectroscopic and analytical data (see Experimental section) are consistent with the proposed structure of the compound, which was confirmed by a single-crystal X-ray diffraction study (Fig. 2).

The structure of $\mathbf{L}$ closely resembles that of its parent (2hydroxyphenyl)bis(pyrazolyl)methane. ${ }^{16}$ molecules of $\mathbf{L}$ consist of two pyrazole rings, oriented in a roughly antiparallel manner with respect to each other, N-bonded to the methine carbon. The 2-propargyloxyphenyl group is attached to the bis(pyrazolyl)methane unit and rotated by $c a$. $30^{\circ}$ out of the 
Table 1 Crystallographic and refinement details for $\mathbf{L},\left[\mathrm{CoCl}_{2}(\mathbf{L})\right]_{2}$ and $\left[\mathrm{ZnCl}_{2}(\mathbf{L})\right]$

\begin{tabular}{|c|c|c|c|}
\hline & $\mathbf{L}$ & {$\left[\mathrm{CoCl}_{2}(\mathbf{L})\right]_{2}$} & {$\left[\mathrm{ZnCl}_{2}(\mathbf{L})\right]$} \\
\hline Formula & $\mathrm{C}_{16} \mathrm{H}_{14} \mathrm{~N}_{4} \mathrm{O}$ & $\mathrm{C}_{16} \mathrm{H}_{14} \mathrm{Cl}_{2} \mathrm{CoN}_{4} \mathrm{O}$ & $\mathrm{C}_{16} \mathrm{H}_{14} \mathrm{Cl}_{2} \mathrm{~N}_{4} \mathrm{OZn}$ \\
\hline$M_{\mathrm{r}}$ & 278.31 & 408.14 & 414.58 \\
\hline Crystal size/mm & $0.07 \times 0.12 \times 0.15$ & $0.07 \times 0.11 \times 0.13$ & $0.09 \times 0.13 \times 0.37$ \\
\hline Crystal system & Monoclinic & Triclinic & Triclinic \\
\hline Space group & $P 2_{1}$ & $P \overline{1}$ & $P \overline{1}$ \\
\hline$a / \AA$ & $9.2557(3)$ & $8.2354(9)$ & $8.1822(4)$ \\
\hline$\alpha /^{\circ}$ & 90 & $95.643(2)$ & $96.5040(10)$ \\
\hline$\beta /^{\circ}$ & $95.411(3)$ & $97.344(2)$ & $95.6410(10)$ \\
\hline$\gamma /{ }^{\circ}$ & 90 & $108.028(2)$ & $103.3830(10)$ \\
\hline$U / \AA^{3}$ & $691.57(4)$ & $829.60(16)$ & $843.18(8)$ \\
\hline$Z$ & 2 & 2 & 2 \\
\hline$D_{\mathrm{c}} / \mathrm{g} \mathrm{cm}^{3}$ & 1.337 & 1.634 & 1.633 \\
\hline No. obs. data $[I>2 \sigma(I)]$ & 3861 & 3851 & 4038 \\
\hline No. parameters/restraints & $190 / 1$ & $273 / 0$ & $273 / 0$ \\
\hline$R_{1}$ (obs. data) & 0.0328 & 0.0462 & 0.0251 \\
\hline$w R_{2}$ (all data) & 0.0878 & 0.0832 & 0.0650 \\
\hline$S$ & 0.974 & 0.864 & 1.060 \\
\hline Min., $\max . \Delta \rho / \mathrm{e} \AA^{-3}$ & $0.293,-0.269$ & $0.674,-0.481$ & $0.459,-0.228$ \\
\hline
\end{tabular}

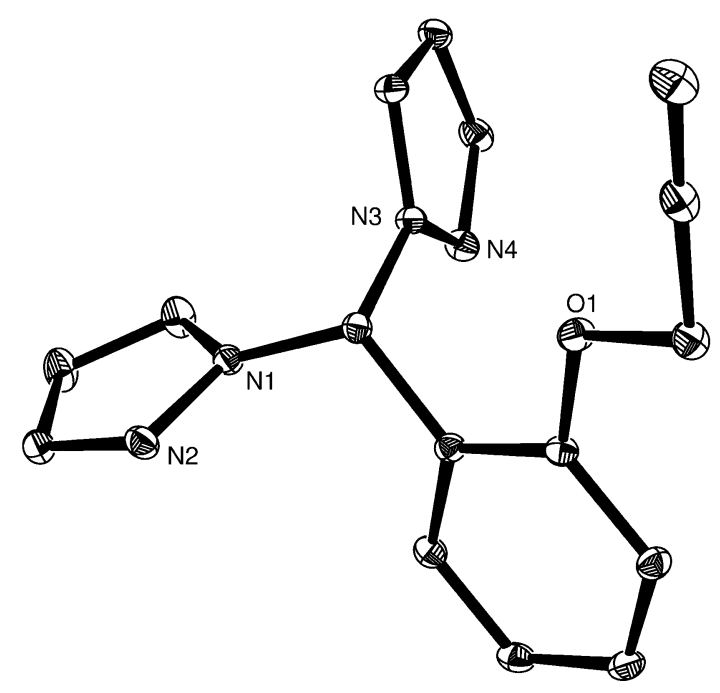

Fig. 2 Molecular structure of $\mathbf{L}$. Ellipsoids show 30\% probability levels and hydrogen atoms have been omitted for clarity.

plane perpendicular to that defined by the $\mathrm{N}-\mathrm{C}-\mathrm{N}$ fragment. Unlike its phenolic precursor or the $\left[\mathrm{CoCl}_{2}(\mathbf{L})\right]_{2}$ complex (see below), molecules of $\mathbf{L}$ display no intramolecular hydrogen bonding contacts or $\pi$-stacking interactions.

\section{Coordination compounds of $\mathbf{L}$}

Initially we examined the behaviour of $\mathbf{L}$ as a bidentate $\mathrm{N}$-donor ligand by preparing some $\mathrm{Ag}(\mathrm{I}), \mathrm{Cu}(\mathrm{II}), \mathrm{Ni}(\mathrm{II}), \mathrm{Co}(\mathrm{II}), \mathrm{Zn}$ (II) and $\mathrm{Pd}(\mathrm{II})$ complexes. With exception of the $\mathrm{Pd}(\mathrm{II})$ derivatives (see below), the M(II) complexes were easily prepared in good yields, by simply mixing ethanolic solutions containing appropriate amounts of $\mathbf{L}$ and the metal salts (Scheme 2).

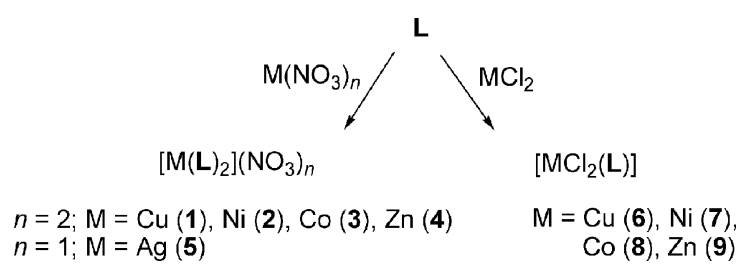

Scheme 2

The compounds were characterised by IR spectroscopy, mass spectrometry and, where possible, by ${ }^{1} \mathrm{H}$ NMR spectroscopy. All of the dichloro compounds, except the $\mathrm{Zn}$ (II) derivative, were poorly soluble in common organic solvents, suggesting the possibility of a dimeric or polymeric structure. This was indeed confirmed by an X-ray diffraction study of $\left[\mathrm{CoCl}_{2} \mathbf{L}\right]_{2}$ (7) and $\left[\mathrm{ZnCl}_{2} \mathbf{L}\right](\mathbf{9})$. The structure of the bright blue, five-coordinate Co(II) complex (Fig. 3) consists of a chloro-bridged dimer of $\mathrm{MCl}_{2} \mathbf{L}$ units.

The coordination about the metal atoms can be described as distorted trigonal bipyramidal. The configuration of the sixmembered ring formed by the bis(pyrazolyl)methane and the Co centre has a boat configuration with the methine proton in an equatorial position. One of the ligand nitrogen atoms is trans to one of the bridging chlorides, whilst the other nitrogen is trans to the non-bridging $\mathrm{Cl}^{-}$ligand. The $\mathrm{Co}-\mathrm{N}$ distances [2.037(3) and 2.124(3) ̊] are quite asymmetric. In the structurally related cobalt(II) dimer [ $\mathrm{CoCl}_{2}\{$ di(2-pyridyl)methoxymethanol $\left.\}\right]_{2}$, obtained as a byproduct of ligand degradation, the $\mathrm{Co}-\mathrm{N}$ distances are 2.119(3) and 2.124(3) A, respectively. ${ }^{23}$ Similarly, the $\mathrm{Co}-\mathrm{Cl}_{\text {bridging }}$ bond lengths in $\left[\mathrm{CoCl}_{2}(\mathbf{L})\right]_{2}$ of 2.3303(9) and 2.6343(9) $\AA$ are much more asymmetric than those in $\left[\mathrm{CoCl}_{2}\{\operatorname{di}(2-\right.$ pyridyl)methoxymethanol $\}]_{2}[2.416(1)$ and 2.438(1) $\AA]$. Furthermore, the propargyloxyphenyl groups are rotated such a way that the alkyne cannot interact with the metal centre. However, 


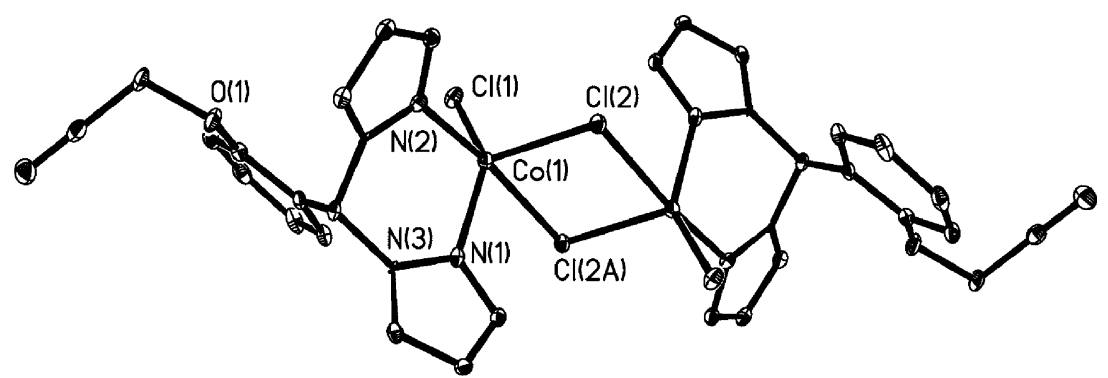

Fig. 3 Molecular structure of $\left[\mathrm{CoCl}_{2} \mathbf{L}\right]_{2}$. Ellipsoids show 30\% probability levels and hydrogen atoms omitted for clarity.

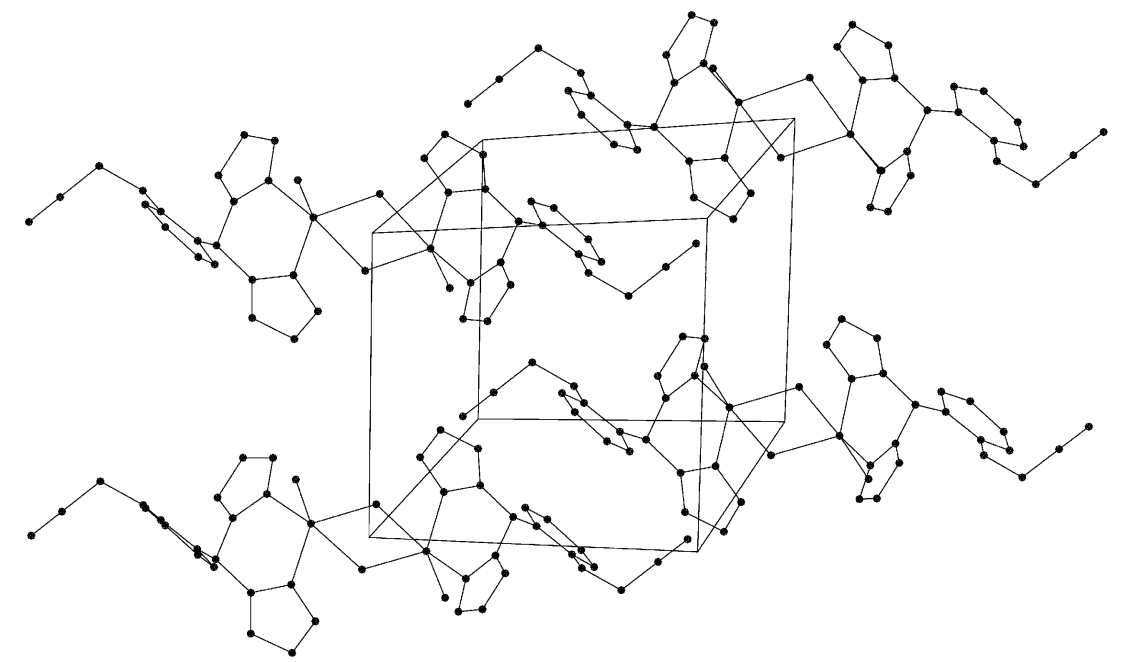

Fig. 4 Packing of $\left[\mathrm{CoCl}_{2} \mathbf{L}\right]_{2}$ showing the phenyl-phenyl interactions between adjacent dimmers.

there are $\pi$-stacking interactions of $c a .3 .8 \AA$ between the propargyloxyphenyl groups of adjacent dimer molecules resulting in a polymeric chain as shown in Fig. 4.

The structure of the $\mathrm{Zn}$ (II) derivative (Fig. 5) contains only monomeric $\left[\mathrm{ZnCl}_{2} \mathbf{L}\right]$ units; $\mathrm{Cl}$ atoms of adjacent molecules are more than $3 \AA$ apart. Like the Co(II) derivative described above, $\left[\mathrm{ZnCl}_{2} \mathbf{L}\right]$ contains the bis(pyrazolyl)methane ligand in a boat conformation. The $\mathrm{Zn}-\mathrm{N}$ distances [2.0515(13) and 2.0920(13) $\AA]$ are similar, but less symmetric, compared to those found in the only other structurally authenticated $\mathrm{Zn}(\mathrm{II}) \mathrm{Cl}_{2}$ complex containing a bis(pyrazolyl)methane ligand $\left[\mathrm{ZnCl}_{2}\left\{\mathrm{Me}_{2} \mathrm{C}(\mathrm{Pz})_{2}\right\}\right]$ [2.058(3) and 2.046(3) $\AA$ ]. ${ }^{24}$ The $\mathrm{Zn}-\mathrm{Cl}$ distances in $\left[\mathrm{ZnCl}_{2} \mathbf{L}\right]$

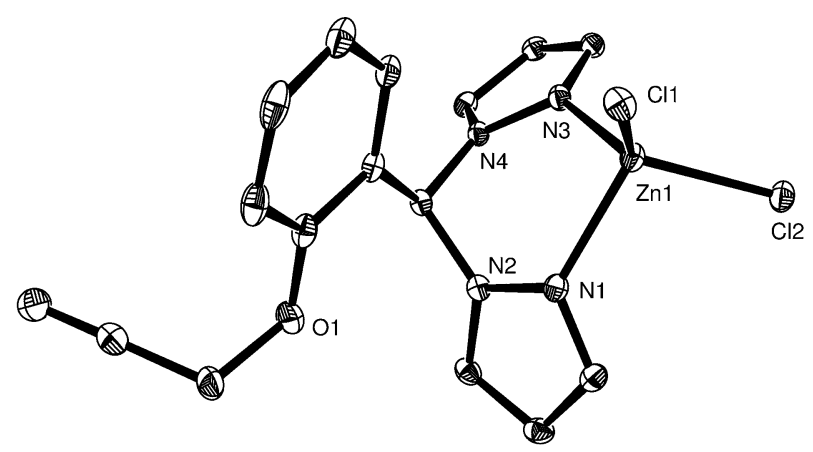

Fig. 5 Molecular structure of $\left[\mathrm{ZnCl}_{2} \mathbf{L}\right]$. Ellipsoids show $40 \%$ probability levels and hydrogen atoms omitted for clarity.
[2.2108(4) and 2.2448(4) $\AA$ ] are also similar to those reported for $\left[\mathrm{ZnCl}_{2}\left\{\mathrm{Me}_{2} \mathrm{C}(\mathrm{Pz})_{2}\right\}\right][2.209(1)$ and 2.229(1) $\mathrm{A}]$.

Curiously, our attempts to prepare $\left[\mathrm{PdCl}_{2}(\mathbf{L})\right]$ by displacement of the weakly coordinated ligands from $\left[\mathrm{PdCl}_{2}(\mathrm{cod})\right]$ or $\left[\mathrm{PdCl}_{2}(\mathrm{NCMe})_{2}\right]$ failed; even after $c a .18 \mathrm{~h}$ reflux only unreacted starting materials were recovered. However, the reaction of $\left[\mathrm{Pd}(\mathrm{OTf})_{2}(\mathrm{dppe})\right]$ with $\mathbf{L}$ gave high yields of the dicationic complex $[\operatorname{Pd}(\mathrm{dppe})(\mathbf{L})](\mathrm{OTf})_{2}(\mathbf{1 0})$. The spectral data of 10 (see Experimental section) are fully consistent with the proposed structure, particularly informative is the ES mass spectrum, the isotopic distribution of which matches perfectly with that computed for the doubly charged $[\operatorname{Pd}(\mathrm{dppe})(\mathbf{L})]^{2+}$ cation. Attempts to deprotonate the alkyne of the coordinated ligand to form alkynyl complexes with other metals only led to decomposition or insoluble materials. Furthermore, the poor solubility of many of the $[\mathrm{ML}]$ derivatives described here hampered further reactions.

\section{$\mathrm{L}$ as $\mathrm{C}$-coordinating ligand}

Treatment of $\mathbf{L}$ with $\left[\mathrm{AuCl}\left(\mathrm{PPh}_{3}\right)\right]$ in the presence of base gives the organometallic gold(I) alkynyl complex $\left[\mathrm{Pz}_{2} \mathrm{CH}\left(\mathrm{C}_{6} \mathrm{H}_{4}-\right.\right.$ 2- $\left.\left.\mathrm{OCH}_{2} \mathrm{CCAuPPh}_{3}\right)\right](\mathbf{1 1})$ in high yield (Scheme 3).

The proton NMR spectrum of the compound shows a singlet resonance due to the $\mathrm{OCH}_{2}$ group, indicating loss of the acetylenic proton, which is further confirmed by the absence of a $\equiv \mathrm{C}-\mathrm{H}$ band in the IR spectrum of the complex. The FAB mass spectrum shows a weak signal for the molecular ion peak, the isotope 


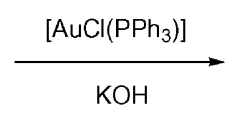<smiles>C(#Cc1ccccc1)COc1ccccc1C(n1cccn1)n1cccn1</smiles>

(11)

Scheme 3

pattern of which is in excellent agreement with the calculated isotope distribution for the complex. Similarly, the reaction of two equivalents of $\mathbf{L}$ with trans- $\left[\mathrm{PtCl}_{2}\left(\mathrm{PPh}_{3}\right)_{2}\right]$ in the presence of $\mathrm{Et}_{2} \mathrm{NH}$ and a catalytic amount of $\mathrm{CuI}$ gives the bis(alkynyl)Pt(II) complex in good yield (Scheme 4).

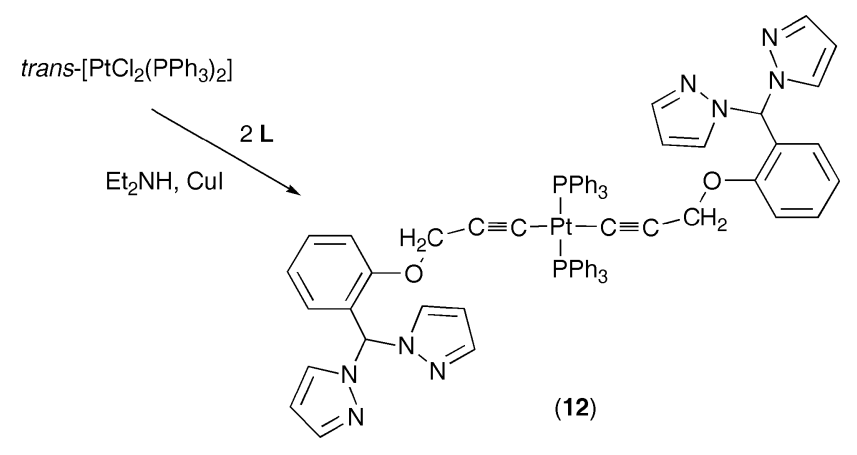

Scheme 4

Again, the ${ }^{1} \mathrm{H}$ NMR and IR spectra clearly indicate Ccoordination of the ligand since no signals due to the $\mathrm{C} \equiv \mathrm{CH}$ group are observed. The ${ }^{31} \mathrm{P}\left\{{ }^{1} \mathrm{H}\right\}$ NMR spectrum of the complex shows a singlet resonance with Pt satellites of $2636 \mathrm{~Hz}$, indicating a trans geometry about the platinum. Complexes $\mathbf{1 1}$ and $\mathbf{1 2}$ still possess the vacant bis(pyrazolyl) unit, which should allow coordination to other metals. To test this hypothesis we studied the reaction of $\mathbf{1 1}$ and $\mathbf{1 2}$ with $\left[\mathrm{Cu}(\mathrm{MeCN})_{4}\right] \mathrm{PF}_{6}$ by electrospray mass spectrometry. In both cases we observed signals, with isotope patterns corresponding to the cationic mixed metal complexes $[11 \mathrm{Cu}]^{+}$and $\left[12 \mathrm{Cu}_{2}\right]^{2+}$, respectively (Fig. 6).

Based on these findings, we then carried out the reaction of complexes $\mathbf{1 1}$ and $\mathbf{1 2}$ with appropriate amounts of $\left[\mathrm{Pd}(\mathrm{OTf})_{2}(\mathrm{dppe})\right]$ and were able to isolate the mixed-metal complexes $\left[(\mathrm{dppe}) \mathrm{PdPz}_{2} \mathrm{CH}\left(\mathrm{C}_{6} \mathrm{H}_{4}-2-\mathrm{OCH}_{2} \mathrm{CCAuPPh}_{3}\right)\right](\mathrm{OTf})_{2}$ (13) and trans-[\{(dppe) $\left.\left.\mathrm{PdPz}_{2} \mathrm{CHC}_{6} \mathrm{H}_{4}-2-\mathrm{OCH}_{2} \mathrm{CC}\right\}_{2} \mathrm{Pt}\left(\mathrm{PPh}_{3}\right)_{2}\right](\mathrm{OTf})_{4}$ (14) in good yields (Scheme 5). These complexes were characterised by NMR spectroscopy and ES mass spectrometry. The former clearly are consistent with the proposed structures, in particular the ${ }^{31} \mathrm{P}\left\{{ }^{1} \mathrm{H}\right\}$ NMR spectra shows two distinct signals corresponding to the dppe and $\mathrm{PPh}_{3}$ phosphorus atoms, respectively. In the case of 14 platinum satellites $\left(J_{\mathrm{Pt} \mathrm{P}}=2620 \mathrm{~Hz}\right)$, typical for trans platinum(II) complexes, are observed. Unfortunately, the ES mass spectra were not very informative as many unassignable fragments containing $\mathrm{Pd} / \mathrm{Au}$ and $\mathrm{Pd} / \mathrm{Pt}$ with various charges could be observed. No molecular ion peaks were detected under various different instrument conditions.

\section{Conclusions}

We have shown here that the new scorpionate (2-propargyloxyphenyl)bis(pyrazolyl)methane is a versatile ligand that can form simple coordination compounds via the nitrogen atoms of the bis(pyrazolyl) unit as well as organometallic complexes through $\mathrm{C}$-coordination of the alkyne functionality. Furthermore, we have demonstrated that both of these coordination modes can operate at the same time to form heterobimetallic $\mathrm{Au} / \mathrm{Pd}, \mathrm{Cu} / \mathrm{Pd}$ and $\mathrm{Pt} / \mathrm{Pd}$ complexes.

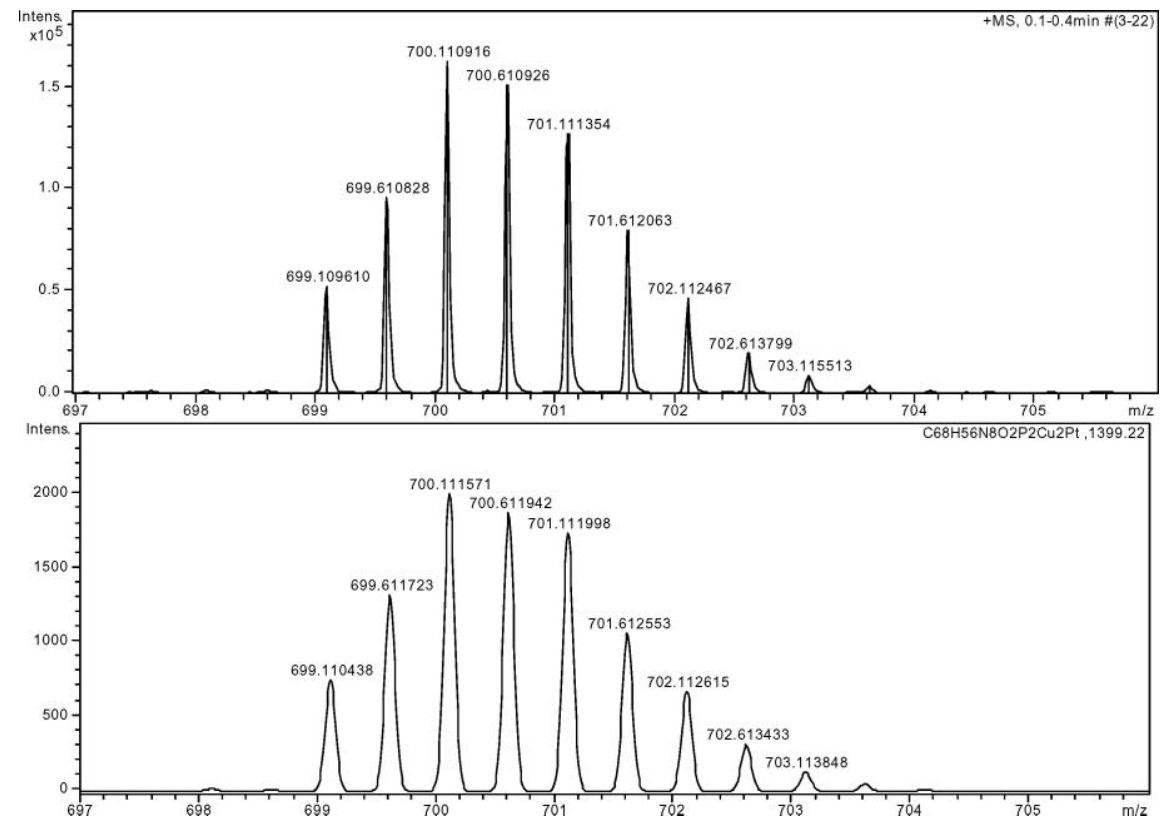

Fig. 6 HR ES mass spectrum of $[\mathbf{1 2 C u}]^{2+}$ (top), computed isotope pattern (bottom). 


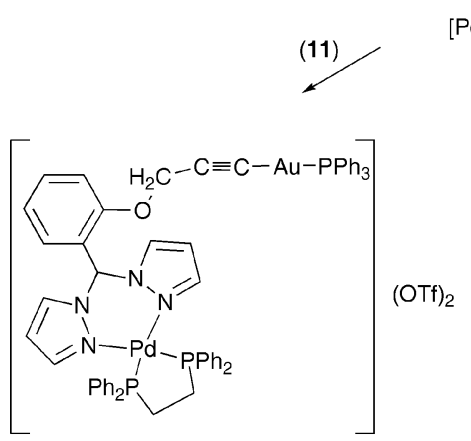

(13)
$\left[\mathrm{Pd}(\mathrm{OTf})_{2}(\mathrm{dppe})\right]$
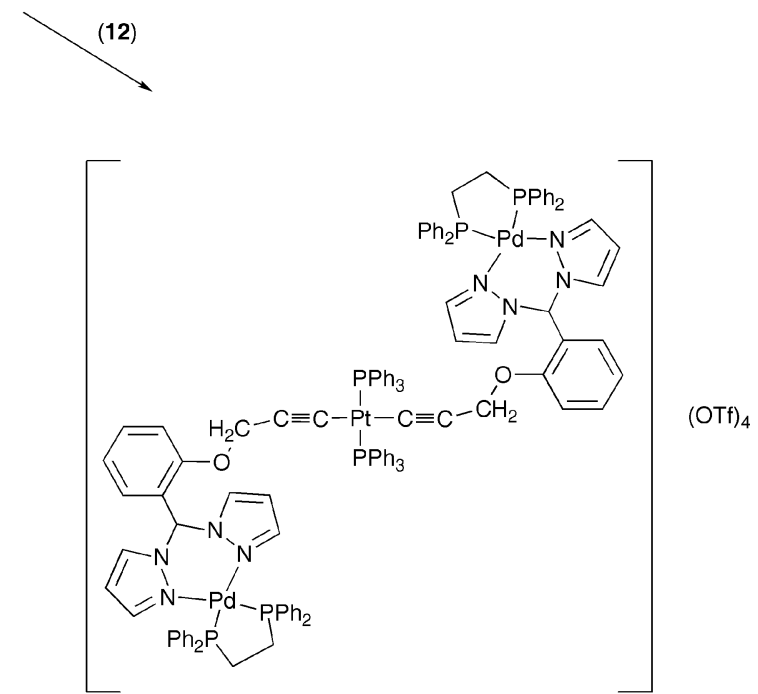

(14)

Scheme 5

\section{References}

1 S. Trofimenko, Chem. Rev., 1993, 93, 943-980.

2 S. Trofimenko, Scorpionates, The Coordination Chemistry of Polypyrazolylborate Ligands, Imperial College Press, London, 1999.

3 S. Trofimenko, Polyhedron, 2004, 23, 197-203.

4 F. T. Edelmann, Angew. Chem., Int. Ed., 2001, 40, 1656-1660.

5 C. Pettinari and C. Santini, in Comprehensive Coordination Chemistry II, ed. J. A. McCleverty and T. J. Meyer, Elsevier, New York, 2004, vol. 1, pp. 159-210.

6 H. R. Bigmore, S. C. Lawrence, P. Mountford and C. S. Tredget, Dalton Trans., 2005, 635-651.

7 C. Pettinari and R. Pettinari, Coord. Chem. Rev., 2005, 249, 525-543.

8 C. Pettinari and R. Pettinari, Coord. Chem. Rev., 2005, 249, 663-691.

9 A. Otero, J. Fernández-Baeza, A. Antiñolo and A. Lara-Sánchez, Dalton Trans., 2004, 1499-1510.

10 R. Usón, A. Laguna, M. Laguna, B. R. Manzano, P. G. Jones and G. M. Sheldrick, J. Chem. Soc., Dalton Trans., 1984, 285-292.

11 A. Luquin, C. Bariain, E. Vergara, E. Cerrada, J. Garrido, I. R. Matias and M. Laguna, Appl. Organomet. Chem., 2005, 19, 1232-1238.

12 L. A. Méndez, J. Jiménez, E. Cerrada, F. Mohr and M. Laguna, J. Am. Chem. Soc., 2005, 127, 852-853.
13 F. Mohr, E. Cerrada and M. Laguna, Organometallics, 2006, 25, 644 648

14 M. I. Bruce, B. K. Nicholson and O. B. Shawkataly, Inorg. Synth., 1989, 26, 324.

15 S. Fallis, G. K. Anderson and N. P. Rath, Organometallics, 1991, 10, $3180-3184$

16 T. C. Higgs and C. J. Carrano, Inorg. Chem., 1997, 36, 291-297.

17 P. K. Byers, A. J. Canty and R. T. Honeyman, J. Organomet. Chem., 1990, 385, 417-427.

18 H. Eckert and B. Forster, Angew. Chem., Int. Ed. Engl., 1987, 26, 894 894

19 CrysAlis CCD and CrysAlis RED: V 1.171.27p8, Oxford Diffraction, Ltd, Oxford, UK, 1995-2005.

20 SAINT version 6.028, Bruker AXS,Madison, WI, 2001.

21 G. M. Sheldrick, SADABS version 2.03, Universität Göttingen, 2002

22 G. M. Sheldrick, SHELXTL-NT 6.1, Universität Göttingen, 1998.

23 C. Hemmert, M. Renz, H. Gornitzka, S. Soulet and B. Meunier, Chem. Eur. J., 1999, 5, 1766-1774.

24 B. Bovio, A. Cingolani and F. Bonati, Z. Anorg. Allg. Chem., 1992, 610, 151-156. 